\title{
Autor: Influência do índice apoptótico e da imuno-expressão \\ da survivina no prognóstico de pacientes com mola hidatiforme completa
}

ANTÔNIO RodriGUes BRAGa Neto

ORIENTADORA:

Marilza Vieira CunHa RudGe

CO-ORIENTADORA:

IZILDINHA MAESTÁ

Maria ApareCida Custódio DominGues

\author{
Influence of the apoptotic index and survivin immuno-expression on the prognosis
} of patients with complete bydatidiform mole

\section{Resumo de tese}

Palavras-chave

Mola hidatiforme completa

Prognóstico

Índice apoptótico

Imuno-expressão da survivina

Keywords:

Complete hydatidiform mole

Prognosis

Apoptotic index

Survivin immuno-expression
Tese apresentada ao Programa de Pós-graduação em Ginecologia, Obstetrícia e Mastologia da Faculdade de Medicina de Botucatu-UNESP, Área de Concentração: Obstetrícia, para obtenção do título de Doutor, em 01 de setembro de 2009.

OBJETIVO: Avaliar a influência do índice apoptótico e da imuno-expressão da survivina em tecido molar no prognóstico de pacientes com mola hidatiforme completa (MHC). MÉTODOS: Estudo observacional, retrospectivo, incluindo 78 pacientes com MHC que realizaram tratamento e seguimento, por pelo menos um ano, no Centro de Doenças Trofoblásticas de Botucatu/SP, entre 1995 e 2006. Baseado nas curvas de regressão da gonadotrofina coriônica, as pacientes foram divididas em dois grupos: remissão espontânea (MHC-RE - 59 pacientes) e evolução para NTG pós-molar (MHC-NTG - 19 pacientes). Avaliação imunohistoquímica de trofoblasto viloso do tecido molar foi realizada pela técnica da avidina-biotina-peroxidase, usando dois marcadores: anticorpo policlonal anti-caspase-3 e anticorpo monoclonal anti-survivina. $\bigcirc$ índice apoptótico foi expresso em porcentual e imuno-expressão da survivina foi avaliada pela determinação de um escore. RESULTADOS: Foi significativo o efeito do índice apoptótico sobre a evolução de pacientes com $\mathrm{MHC}$, de tal modo que, o aumento de 1 unidade no índice apoptótico reduziu, em média, $61 \%$ a chance de desenvolvimento de NTG pós-molar $(O R=0,61,95 \%$ IC: 0,45-0,84). Não houve influência significativa da imuno-expressão da survivina no desenvolvimento de NTG pós-molar (p>0,05; teste exato de Fisher). CONCLUSÃO: Neste estudo, o índice apoptótico foi bom preditor do desenvolvimento de NTG depois de MHC, com potencial para ser usado como biomarcador prognóstico dessa doença. Por outro lado, a imuno-expressão da survivina no trofoblasto viloso não influenciou a evolução da MHC para NTG pós-molar. 